Polymer Journal, Vol. 2, No. 4, pp 445-456 (1971)

\title{
A Hole Theory of Polymer Liquids and Glasses. IV. Glass Transition under Elevated Pressure and Densified Glasses
}

\author{
Takuhei Nose \\ Department of Polymer Technology, \\ Tokyo Instituie of Technology, Tokyo, Japan.
}

(Received July 6, 1970)

\begin{abstract}
The pressure dependence of the glass transition temperature $\left(\partial T_{\mathrm{g}} / \partial P\right)$ and the enthalpy of the densified glass were studied with a hole theory. It was found that not the assertion of the iso-free volume but that of the iso-configurational entropy (or energy) or Adam-Gibbs theory at the glass transition is supported by the observed fact $\left(\partial T_{\mathrm{g}} / \partial P\right) \cong T V \Delta \alpha / \Delta C_{p}<\Delta \beta / \Delta \alpha$, where $\Delta \alpha, \Delta \beta$, and $\Delta C_{p}$ are the differences in thermal expansion coefficient, compressibility, and heat capacity at constant pressure between the liquid and glassy states at $T_{\mathrm{g}}$, respectively. It is also supported by the fact that the densified glass has almost the same enthalpy as the glass obtained under ordinary conditions. The inequality of $\left(\partial T_{\mathrm{g}} / \partial P\right)$ and $\Delta \beta / \Delta \alpha$, which causes the densification of the glass formed under elevated pressure, was quantitatively related to the magnitute of the densified volume. It is suggested that the inequality $T V \Delta \alpha / \Delta C_{p}<\Delta \beta / \Delta \alpha$ and the formation of the densified glass may be closely related to intrasegmental interactions or the chain conformation.
\end{abstract}

KEY WORDS Hole Theory / Polymer / Glass Transition / Pressure /

Free Volume / Configurational Entropy / Adam-Gibbs Theory /

Densified Glass / Annealed Glass /

Recently many investigations have been made on the pressure dependence of various physical properties of polymers, affording significant information particularly on glass trsnsition and the glassy state. Noticeable results from such investigations are: (1) the pressure dependence of the glass transition temperature $T_{\mathrm{g}}$ is related to thermodynamic quantities by the equation $\left(\partial T_{\mathrm{g}} / \partial P\right) \cong T V \Delta \alpha / \Delta C_{p}<\Delta \beta / \Delta \alpha,{ }^{1,2}$ where $P, T$, and $V$ are pressure, absolute temperature, and volume, respectively, and $\Delta \beta, \Delta \alpha$, and $\Delta C_{p}$ are the differences in compressibility, thermal expansion coefficient, and heat capacity at constant pressure between the liquid and glassy states, respectively; (2) one can obtain a densified glass by glass-forming under elevated pressure, $;^{3,4}$ and (3) the magnitude of densified volume is directly and quantitatively related to the the inequality of $\left(\partial T_{\mathrm{g}} / \partial P\right)$ and $\Delta \beta / \Delta \alpha .^{4}$ As O'Reilly ${ }^{1}$ and Goldstein ${ }^{2}$ have pointed out, (1) above appears to show that not the volume, but the entropy or the enthalpy plays an important role in the glass transition.

Today there are two main theories of glass transition: one is the well known iso-free volume theory, ${ }^{5}$ and the other is the theory presented by Gibbs, DiMerzio, and Adam. ${ }^{6,7}$ According to the latter theory, a thermodynamic secondorder transition should occur when the configurational entropy becomes zero ${ }^{6}$ and the glass transition observed is higher than the second-order transition temperature by a definite number of degrees (about $50^{\circ} \mathrm{C}$ ). ${ }^{7}$ This theory ${ }^{7}$ also shows that the temperature dependence of the relaxation time of segmental motion is determined by the product of absolute temperature and the configurational entropy of a system. The observed fact (1) appears to support the second assertion.

In this paper, from our hole theory preposed previously ${ }^{8}$ we derive thermodynamic relations for the change in glass transition temperature with pressure and the enthalpies of the densified and annealed glasses. Comparing experimental 
results with the theoretical ones we examine the validity of the above-mentioned assertions for glass transition. We can show that the relation $\left(\partial T_{\mathrm{g}} / \partial P\right)=T V \Delta \alpha / \Delta C_{p}$ and no difference in enthalpy between densified glasses formed under different pressures imply that the glass transition occurs at constant configurational entropy (or energy) within a substance. We also quantitatively relate the inequality between $\left(\partial T_{\mathrm{g}} / \partial P\right)$ and $\Delta \beta / \Delta \alpha$ to the magnitude of densified volume and suggest that the inequality is closely related to the conformational degrees of freedom of a chain backbone.

\section{THEORY}

\section{Model}

First we describe the hole model presented in the preceding paper, ${ }^{8}$ which is adopted here with a new assumption. We consider a system in which $N$ segments are distributed on $M$ lattice sites $(M \geq N)$. Thus there are $M-N$ empty sites or holes. If the cell volume is denoted by $v^{*}$, then $M / N=V /\left(N v^{*}\right) \equiv V / V^{*} \equiv \tilde{V}$, and therefore the quantity $\left(1-\tilde{V}^{-1}\right)$ represents the hole fraction. To develop the theory further, we now make the following two assumptions.

Assumption 1. The change in cell volume with pressure and temperature is essentially independent of the hole fraction or $\tilde{V}$. This implies that the thermal expansion coefficient and compressibility of the cell volume are almost continuous at the glass transition, and that the glasses of different hole fractions have almost the same cell volume, the same thermal expansion coefficient, and the same compressibility.

Assumption 2. The free energy of the system is expressed by the sum of two kinds of free energy; one associated with the internal degrees of freedom or intrasegmental interactions, including the so-called short range interaction related to the chain conformation, and the other associated with the external degrees of freedom or intersegmental interactions. Namely, the free energy $F$ is written

$$
\begin{aligned}
F= & F_{1}(T)+F_{2}\left(\tilde{V}, V^{*}, T\right)=U_{1}(T)-T S_{1}(T) \\
& +U_{2}\left(\tilde{V}, V^{*}, T\right)-T S_{2}\left(\tilde{V}, V^{*}, T\right)
\end{aligned}
$$

where the the subscripts 1 and 2 refer to intraand inter-segmental interactions, respectively, and $U$ is the internal energy and $S$ the entropy.

The glassy state is considered here to be a quasi-equilibrium state, and is regarded as a frozen liquid in which both holes and chain conformation are frozen-in. Thus, in the glassy state, the hole fraction or $\tilde{V}$ is assumed to be constant and a part of $F_{1}(T)$ associated with the hindered rotaion about chain backbones is also assumed not to change with temperature and pressure.

In the liquid state, two of the three variables $\tilde{V}, V^{*}$, and $T$ in eq 1 are independent and these variables change so as to minimize the free energy, i.e., $\left(\partial F / \partial V^{*}\right)_{V, T}=0$. Hence, from this condition $\left(\partial F / \partial V^{*}\right)_{V, T}=0$ and a thermodynamic equation $P=-(\partial F / \partial V)_{T}$, we have

$$
P \tilde{V}+\left(\partial F_{2} / \partial V^{*}\right) \tilde{V}, T=0
$$

and

$$
P V^{*}+\left(\partial F_{2} / \partial \tilde{V}\right)_{V^{*}, T}=0
$$

which are the equation of state in the liquid state (see Appendix in ref 9). On the other hand, the equation of state in the glassy state can be expressed by

$$
P \tilde{V}_{\mathrm{g}}+\left(\partial F_{2} / \partial V^{*}\right) \tilde{\mathrm{V}, T}=0
$$

which is derived from $P=-(\partial F / \partial V)_{T}$ with $\tilde{V}=$ $\widetilde{V}_{g}=$ constant. It is noticed here that the equation of state in the glassy state, eq 4 , and eq 2 have the same form except that $\tilde{V}$ can be variable or not. Accordingly, Assumption 1 implies that $\left(\partial F_{2} / \partial V^{*}\right)_{\tilde{V}, T}$ is approximately a function of $V^{*}$ and $T$. In other words, Assumption 1 suggests that $F_{2}$ is approximated by the form

$$
\begin{aligned}
F_{2}\left(\tilde{V}, V^{*} T\right)= & F_{2}{ }^{\prime}(\tilde{V}, T)+F_{2}{ }^{\prime \prime}\left(V^{*}, T\right) \\
= & U_{2}{ }^{\prime}(\tilde{V}, T)-T S_{2}{ }^{\prime}(\tilde{V}, T) \\
& +U_{2}{ }^{\prime \prime}\left(V^{*}, T\right)-T S_{2}{ }^{\prime \prime}\left(V^{*}, T\right)
\end{aligned}
$$

(see Appendix).

In the preceding paper, ${ }^{8}$ from the hole model described above, we derived the relations

$$
\begin{aligned}
& T V(\Delta \alpha)^{2} /\left(\Delta C_{p}^{\text {inter }} \Delta \beta\right)=1 \geq T V(\Delta \alpha)^{2} /\left(\Delta C_{p} \Delta \beta\right) \\
& \Delta C_{p}=\Delta C_{p}^{\text {inter }}+\Delta C_{p}^{\text {intra }} \\
& \text { and } \begin{array}{c}
\left(\partial S_{2} / \partial \tilde{V}\right)_{V^{*}, T}=V^{*} \Delta \alpha / \Delta \beta
\end{array}
\end{aligned}
$$$$
\text { and }
$$

where the superscripts inter and intra refer re- 
spectively to inter- and intra-segmental interactions.

In this paper we add the following assumption, Assumption 3, to the above two assumptions in order to define the configurational entropy $S^{\mathrm{c}}$ as the entropy which is frozen-in in the glassy state, in other words, as the entropy determined by the hole fraction, or $\tilde{V}$, and the state of chain conformation.

Assumption 3. The heat capacity of the glass is independent of the frozen hole fraction or $\tilde{V}_{\mathrm{g}}$. This assumption implies that $\left[\partial\left(\partial S_{2} / \partial T\right) \check{V} / \partial \tilde{V}\right]_{V^{*}, T}$ $=\left[\partial\left(\partial S_{2} / \partial \tilde{V}\right)_{V^{*}, T} / \partial T\right]_{\tilde{V}}=\left[\partial\left(\partial S_{2}{ }^{\prime} / \partial \tilde{V}\right)_{V^{*}, T} / \partial T\right]_{\tilde{V}}=0$, i.e., $\left(\partial S_{2} / \partial \tilde{V}\right)_{V^{*}, T}=\left(\partial S_{2}{ }^{\prime} / \partial \tilde{V}\right)_{V^{*}, T}$ is independent of $T$. It follows, therefore, from Assumption 3 and eq 5 derived from Assumptions 1 and 2, that $\left(\partial S_{2} / \partial \tilde{V}\right)_{V^{*}, T}$ is considered to be a function of $\tilde{V}$ only. Therefore we can define the configurational entropy $S^{\mathrm{c}}$ as a function of state which is determined by the frozen variables, $\tilde{V}$ and $S_{1}^{\mathrm{c}}$ :

$$
\mathrm{d} S^{\mathrm{c}}=\left(\partial S_{2} / \partial \tilde{V}\right)_{V^{*}, T} \mathrm{~d} \tilde{V}+\mathrm{d} S_{1}^{\mathrm{c}}
$$

where $S_{1}{ }^{\text {c }}$ is a part of $S_{1}$ associated with the chain conformation. The configurational entropy thus defined consists of two kinds of entropy, i.e., the entropy arising from the hole, in other words intersegmental interactions and the entropy $S_{1}$, both frozen-in in the glassy state as mentioned before.

From eq 6, 7, 8, and 9, and Assumption 1, we have

$$
\mathrm{d} S^{\mathrm{c}}=\left(\Delta C_{p} / T\right) \mathrm{d} T-V \Delta \alpha \mathrm{d} P
$$

for the liquid state. On the other hand, in the glassy state, $S^{\text {c }}$ does not change as seen from the definition of eq 9, i.e., $\mathrm{d} S^{\mathrm{c}}=0$.

Just as $S^{\mathrm{c}}$, the configurational energy $U^{\mathrm{c}}$ can be defined as

$$
\mathrm{d} U^{\mathrm{c}}=\left(\partial U_{2} / \partial \tilde{V}\right)_{V^{*}, T} \mathrm{~d} \tilde{V}+\mathrm{d} U_{1}{ }^{\mathrm{c}}
$$

where $U_{1}^{\text {c }}$ is a part of $U_{1}$ attributable to the chain conformation. From eq 9, 10, and 11, and Assumption 1, we have

$$
\begin{aligned}
\mathrm{d} U^{\mathrm{c}} & =T \mathrm{~d} S^{\mathrm{c}}+P V(-\Delta \alpha \mathrm{d} T+\Delta \beta \mathrm{d} P) \\
& =\left(\Delta C_{p}-P V \Delta \alpha\right) \mathrm{d} T+(-T V \Delta \alpha+P V \Delta \beta) \mathrm{d} P
\end{aligned}
$$

for the liquid state, noting $\mathrm{d} U_{1}{ }^{\mathrm{c}}=T \mathrm{~d} S_{1}{ }^{\mathrm{c}}=$
$\Delta C_{p}^{\text {intra }} \mathrm{d} T$ and

$$
P V^{*}+\left(\partial U_{2} / \partial \tilde{V}\right)_{V^{*}, T}-T\left(\partial S_{2} / \partial \tilde{V}\right)_{V^{*}, T}=0
$$

which is derived from eq 3 . On the other hand, in the glassy state, $\mathrm{d} U^{\mathrm{c}}=0$ as readily seen from the definition of eq 11 .

In contrast with $S^{\mathrm{c}}$ and $U^{\mathrm{c}}$, we cannot reasonablely define the configurational enthalpy $H^{2}$ as a part of enthalpy frozen-in in the glassy state. If we define $H^{c}$ as $\delta H^{c}=\left[\left(\partial U_{2} / \partial \tilde{V}\right)_{V^{*}, T}\right.$ $\left.+P V^{*}\right] \mathrm{d} \tilde{V}+\mathrm{d} U_{1}^{\mathrm{c}}=T \mathrm{~d} S^{\mathrm{c}}$ as we did in the other paper, ${ }^{10}$ we have $\delta H^{c}=0$ when $\mathrm{d} S^{c}=0$. This indicates that $H^{\mathrm{c}}$ is frozen-in in the glassy state and that the concept of iso- $H^{\mathrm{c}}$ is equivalent to that of iso- $S^{\mathrm{c}}$. In this case, however, $H^{\mathrm{c}}$ is the heat arising from change of $S^{\mathrm{c}}$ and is not a function of state. Under low pressure, $P V^{*}$ is negligibly small compared with $\left(\partial U_{2} / \partial \tilde{V}\right)_{V^{*}, T}$ (cf. next section), and $H^{\mathrm{c}}$ becomes approximately a function of state. In such a case, however, $H^{c}$ is equivalent to $U^{\mathrm{c}}$ defined by eq 11 , and accordingly it is unnecessary to define such a quantity $H^{\mathrm{c}}$ in addition to $U^{\mathrm{c}}$. On the other hand, if we define $H^{c}$ as

$$
H^{\mathrm{c}}=U^{\mathrm{c}}+P\left(V-V^{*}\right)
$$

as the excess enthalpy relative to the glass having no hole $\left(V / V^{*}=1\right)$, which is a function of state, it follows that

$$
\begin{aligned}
\mathrm{d} H^{\mathrm{c}}= & T \mathrm{~d} S^{\mathrm{c}}+\left(V-V^{*}\right) \alpha_{\mathrm{g}} P \mathrm{~d} T+\left(V-V^{*}\right)\left(1-\beta_{\mathrm{g}} P\right) \mathrm{d} P \\
= & \Delta C_{p} \mathrm{~d} T+\left(V-V^{*}\right) \alpha_{\mathrm{g}} P \mathrm{~d} T+\{-T V \Delta \alpha \\
& \left.+\left(V-V^{*}\right)\left(1-\beta_{\mathrm{g}} P\right)\right\} \mathrm{d} P \\
\cong & T \mathrm{~d} S^{\mathrm{c}}+\left(V-V^{*}\right) \mathrm{d} P \\
\cong & \Delta C_{p} \mathrm{~d} T+\left\{-T V \Delta \alpha+\left(V-V^{*}\right)\right\} \mathrm{d} P
\end{aligned}
$$

in the liquid state, and

$$
\begin{aligned}
\mathrm{d} H^{\mathrm{c}} & =P V\left(1-\tilde{V}_{\mathrm{g}}^{-1}\right) \alpha_{\mathrm{g}} \mathrm{d} T+V\left(1-\tilde{V}_{\mathrm{g}}^{-1}\right)\left(1-\beta_{\mathrm{g}} P\right) \mathrm{d} P \\
& \cong V\left(1-\tilde{V}_{\mathrm{g}}^{-1}\right) \mathrm{d} P
\end{aligned}
$$

in the glassy state, where $\alpha_{\mathrm{g}}$ and $\beta_{\mathrm{g}}$ are respectively the thermal expansion coefficient and compressiblity of the glass. According to the difinition of eq $14, H^{\mathrm{c}}$ does not only depend upon the liquid structure which is frozen-in in the glassy state (i.e., the hole fraction and the chain conformation), but also upon absolute $T$ and $P$. This leads to the fact that $H^{c}$ is not frozen-in in the glassy state, as seen in eq 17 . In this sense, $H$ is different from $S^{\mathrm{c}}$ and $U^{\mathrm{c}}$ in the definition. 


\section{T. Nose}

In this paper we use the definition provided by eq 14.

\section{Expression for $\left(\partial T_{\mathrm{g}} / \partial P\right)$}

From eq 10, 13, and 15, we obtain

$$
\begin{aligned}
\left(\partial T_{\mathrm{g}} / \partial P\right) \equiv & (\partial T / \partial P)_{\mathrm{g}} \\
= & \left\{T V \Delta \alpha+T\left(\partial S^{\mathrm{c}} / \partial P\right)_{\mathrm{g}}\right\} / \Delta C_{p} \\
= & \{T V \Delta \alpha-P V \Delta \beta \\
& \left.+\left(\partial U^{\mathrm{c}} / \partial P\right)_{\mathrm{g}}\right\} /\left(\Delta C_{p}-P V \Delta \alpha\right) \\
= & \left\{T V \Delta \alpha-\left(V-V^{*}\right)+\left(\partial H^{\mathrm{c}} / \partial P\right)_{\mathrm{g}}\right\} / \Delta C_{p}
\end{aligned}
$$

where the symbol $(\partial x / \partial y)_{g}$ designates the differentiation along the glass transition point, i.e., the variation of $x_{\mathrm{g}}, x$ at glass transition point, with $y$.

On the other hand, since we can derive

$$
\mathrm{d} \ln \tilde{V}=\Delta \alpha \mathrm{d} T-\Delta \beta \mathrm{d} P
$$

in the liquid state from Assumption 1, we have

$$
\begin{aligned}
\left(\partial T_{\mathrm{g}} / \partial P\right) & =\Delta \beta /\left(\Delta \alpha+\alpha^{\prime}\right) \\
& =\left(\Delta \beta-\beta^{\prime}\right) / \Delta \alpha \\
& =\beta^{\prime} / \alpha^{\prime}
\end{aligned}
$$

where

$$
\begin{aligned}
& \alpha^{\prime}=-(\partial \ln \tilde{V} / \partial T)_{g} \\
& \beta^{\prime}=-(\partial \ln \tilde{V} / \partial P)_{\mathrm{g}}
\end{aligned}
$$

The quantities $\alpha^{\prime}$ and $\beta^{\prime}$ denote the decrease in the frozen hole fraction, in other words the densified volume, because the cell volumes of glasses having different $\tilde{V}$ are almost the same according to Assumption 1, i.e.,

$$
\begin{aligned}
\alpha^{\prime} & =-\left(\partial \ln V_{\mathrm{s}} / \partial T_{\mathrm{g}}\right) \\
\beta^{\prime} & =-\left(\partial \ln V_{\mathrm{s}} / \partial P_{\mathrm{g}}\right)
\end{aligned}
$$

where $V_{\mathrm{s}}$ is the volume of the glass formed at $T_{\mathrm{g}}$ and $\boldsymbol{P}_{\mathrm{g}}$ measured at the same temperature and pressure.

\section{Enthalpies of Glasses Formed under Different Conditions}

We will now consider the difference of enthalpy $\Delta H$ between the glasses formed under different conditions compard at a constant state $\left(T_{0}, P_{0}\right)$, where $P_{0}$ is atmospheric pressure. If the glass which was formed at an ordinary cooling rate at atmosphersc pressure, i.e., formed at the state I $\left(T_{g_{0}}, P_{0}, \tilde{V}_{g_{0}}\right)$, is taken as the reference glass (Glass I) and if the other glass (Glass II) was formed at state II $\left(T_{\mathrm{g}}, \boldsymbol{P}_{\mathrm{g}}, \widetilde{V}_{\mathrm{g}}\right)$, we can express the difference in $H, \Delta H$, between the two glasses, using Assumption 1 and 2 with eq 5 , as

$$
\begin{aligned}
\Delta H= & H(\text { Glass II })-H(\text { Glass I }) \\
= & U_{2}{ }^{\prime}\left(\tilde{V}_{\mathrm{g}}, T_{0}\right)-U_{2}{ }^{\prime}\left(\tilde{V}_{\mathrm{g} 0}, T_{0}\right) \\
& +P_{0}\left(\tilde{V}_{\mathrm{g}}-\tilde{V}_{\mathrm{g} 0}\right) V_{0}{ }^{*}+U_{1}^{\mathrm{c}}\left(T_{\mathrm{g}}\right)-U_{1}^{\mathrm{c}}\left(T_{\mathrm{g} 0}\right)
\end{aligned}
$$

where $V_{0}^{*}$ is the cell volume at $\left(T_{0}, P_{0}\right)$ being independent of $\tilde{V}_{\mathrm{g}}$. By the use of Assumption 3 , eq 11,12 , and 16 , eq 28 can be rewritten

$$
\begin{aligned}
H= & \int_{\mathrm{I}}^{\mathrm{II}}\left(\mathrm{d} U^{\mathrm{c}}\right)^{1}+P_{0}\left(\tilde{V}_{\mathrm{g}}-\tilde{V}_{\mathrm{g} 0}\right) V_{0}^{*} \\
= & \int_{\mathrm{I}}^{\mathrm{II}}\left\{T\left(\mathrm{~d} S^{\mathrm{c}}\right)^{1}+P V(-\Delta \alpha \mathrm{d} T+\Delta \beta \mathrm{d} P)\right\} \\
& +\boldsymbol{P}_{0}\left(\tilde{V}_{\mathrm{g}}-\tilde{V}_{\mathrm{g} 0}\right) V_{0}^{*} \\
= & \int_{\mathrm{I}}^{\mathrm{II}}\left[\left(\mathrm{d} H^{\mathrm{c}}\right)^{1}-P V \Delta \alpha \mathrm{d} T-\left\{\left(V-V^{*}\right)\right.\right. \\
& -P V \Delta \beta\} \mathrm{d} P]+P_{0}\left(\tilde{V}_{\mathrm{g}}-\tilde{V}_{\mathrm{g} 0}\right) V_{0}^{*}
\end{aligned}
$$

where $\left(\mathrm{d} X^{\mathrm{c}}\right)^{1}$ designates the change of $X^{\mathrm{c}}$ in the liquid state.

Now we express $\Delta H^{\text {intra }}$ and $\Delta H^{\text {inter }}$ separately, where $\Delta H^{\text {intra }}$ and $\Delta H^{\text {inter }}$ denote the enthalpy differences associated with intra- and inter-segmental interactions respectively. As seen from eq $28, \Delta H^{\text {intra }}$ is expressed as

$$
\begin{aligned}
\Delta H^{\text {intra }} & =U_{1}^{\mathrm{c}}\left(T_{\mathrm{g}}\right)-U_{1}^{\mathrm{c}}\left(T_{\mathrm{g} 0}\right) \\
& =\int_{T_{\mathrm{g} 0}}^{T_{\mathrm{g}}} \Delta C_{p}^{\text {intra }} \mathrm{d} T \\
& =\Delta \bar{C}_{p}^{\text {intra }} \Delta T_{\mathrm{g}}
\end{aligned}
$$

where $\Delta \bar{C}_{p}^{\text {intra }}$ is the mean value of $\Delta C_{p}^{\text {intra }}$ in the temperature range between $T_{\mathrm{g}}$ and $T_{\mathrm{g} 0}$, and $\Delta T_{\mathrm{g}} \equiv T_{\mathrm{g}}-T_{\mathrm{g} 0}$. On the other hand, we have, from eq 11 and 29 with eq $3^{\prime}, 6,7,8$, and 21 ,

$$
\begin{aligned}
\Delta H^{\text {inter }}= & \int_{\tilde{V}_{\mathrm{g} 0}}^{\widetilde{V}_{\mathrm{g}}}\left(\partial U_{2} / \partial \tilde{V}\right)_{V^{*}, T} \mathrm{~d} \ln \tilde{V}+P_{0}\left(V_{\mathrm{g}}-V_{\mathrm{g} 0}\right) \\
= & \int_{\tilde{V}_{\mathrm{g}}}^{\widetilde{V}_{\mathrm{g} 0}}\{T V \Delta \alpha / \Delta \beta-P V\} \mathrm{d} \ln \tilde{V} \\
& +P_{0}\left(\tilde{V}_{\mathrm{g}}-\tilde{V}_{\mathrm{g} 0}\right) V_{0}^{*} \\
= & \int_{\tilde{V}_{\mathrm{g} 0}}^{\widetilde{V}_{\mathrm{g}}}\left\{\Delta C_{p}^{\text {inter }} / \Delta \alpha-P V\right] \mathrm{d} \ln \tilde{V}
\end{aligned}
$$


A Hole Theory of Polymer Liquids and Glasses. IV

$$
\begin{aligned}
& +P_{0}\left(\tilde{V}_{\mathrm{g}}-\tilde{V}_{\mathrm{g} 0}\right) V_{0}^{*} \\
\cong & \int_{\widetilde{V}_{\mathrm{g} 0}}^{\widetilde{V}_{\mathrm{g}}}\left\{\Delta C_{p}^{\text {inter }} / \Delta \alpha-\left(P-P_{0}\right) V\right\} \mathrm{d} \ln \tilde{V}
\end{aligned}
$$

Here, it is noteworthy that the integrals in eq $33-34^{\prime}$ do not depend upon the integrating path, because $\left(\partial U_{2} / \partial \tilde{V}\right)_{V^{*}, T}$ is a function of $\tilde{V}$ only as $\left(\partial S_{2} / \partial \tilde{V}\right)_{V^{*}, T}$ is. Accordingly, if we change $\tilde{V}$ under atmospheric pressure $\boldsymbol{P}=\boldsymbol{P}_{0}$ by altering the temperature, we derive, from eq $34^{\prime}$.

$$
\Delta H^{\text {inter }}=-\Delta \vec{C}_{p}^{\text {inter }}(1-\mathrm{d}) / \Delta \bar{\alpha}
$$

where $\Delta \bar{C}_{p}^{\text {inter }}$ and $\Delta \bar{\alpha}$ are the mean values for $\Delta C_{p}^{\text {inter }}$ and $\Delta \alpha$ in the temperature range between $T_{\mathrm{g} 0}$ and $T_{\mathrm{g} 0}-(1-d) / \Delta \bar{\alpha}$ under atmospheric pressure. The symbol $d$ is defined as $\tilde{V}_{\mathrm{g}} / \tilde{V}_{\mathrm{g} 0}$ which represents the ratio of the specific volume of Glass II to that of Glass I because of Assumption 1 .

By the use of eq 19, 21, and 29 with $1-V^{*} / V_{0}^{*}=O\left(\beta_{\mathrm{g}} P\right) \ll 1$, we can obtain the quantitative relation between $\left(\partial T_{\mathrm{g}} / \partial P\right)$ and $\left(\partial \Delta H / \partial P_{\mathrm{g}}\right)$ as

$$
\begin{aligned}
\left(\partial T_{\mathrm{g}} / \partial P\right)= & \left\{T V \Delta \alpha-\left(P-P_{0}\right) \Delta \beta\right. \\
& \left.+\left(\partial \Delta H / \partial P_{\mathrm{g}}\right)\right\} /\left\{\Delta C_{p}-\left(P-P_{0}\right) V \Delta \alpha\right\}
\end{aligned}
$$

or

$$
\begin{aligned}
\left(\partial \Delta H / \partial P_{\mathrm{g}}\right)= & \left\{\Delta C_{p}-\left(P-P_{0}\right) V \Delta \alpha\right\}\left(\partial T_{\mathrm{g}} / \partial P\right) \\
& -T V \Delta \alpha+\left(P-P_{0}\right) V \Delta \beta
\end{aligned}
$$

Criteria for $T_{\mathrm{g}}$ and Thermodynamical Relations

Iso-Free Volume. If the iso-free volume at $T_{\mathrm{g}}$ is a valid assumption, i.e., $\left(\partial \tilde{V} / \partial T_{\mathrm{g}}\right)=0$, we can derive, from eq $6,7,22$, and 25 ,

$$
\left(\partial T_{\mathrm{g}} / \partial P\right)=\Delta \beta / \Delta \alpha=T V \Delta \alpha / \Delta C_{p}^{\text {inter }} \geq T V \Delta \alpha / \Delta C_{p}
$$

and

$$
\alpha^{\prime}=\beta^{\prime}=0
$$

According to eq 39, the densified glass could not be formed, i.e., $d=1$.

From eq $32^{\prime}$ and 35 with $d=1$, we have

$$
\Delta H=\Delta \bar{C}_{p}^{\text {intra }} \Delta T_{\mathrm{g}}
$$

for the glass formed under elevated pressure. Therefore the glass obtained under pressure should have higher enthalpy when the iso-free volume assumption is satisfied.

Iso-Configurational Entropy. If the assumption of iso-configurational entropy (iso- $S^{\mathrm{c}}$ ) at $T_{\mathrm{g}}$ is valid, i.e., $\left(\partial S^{\mathrm{c}} / \partial P\right)_{\mathrm{g}}=0$, it follows that eq 6 , 18,22 , and 23 yield

$$
\begin{aligned}
\left(\partial T_{\mathrm{g}} / \partial P\right) & =T V \Delta \alpha / \Delta C_{p}=\Delta \beta /\left(\Delta \alpha+\alpha^{\prime}\right) \\
& =\left(\Delta \beta-\beta^{\prime}\right) / \Delta \alpha \leq \Delta \beta / \Delta \alpha
\end{aligned}
$$

Thus, in this case, $\alpha^{\prime}$ and $\beta^{\prime}$ can be expressed, from eq 6,7 , and 41 , as

$$
\alpha^{\prime}=\left\{\frac{\Delta C_{p} \Delta \beta}{T V(\Delta \alpha)^{2}}-1\right\} \Delta \alpha=\left(\Delta C_{p}^{\text {intra }} / \Delta C_{p}^{\text {inter }}\right) \Delta \alpha \geq 0
$$

and

$$
\beta^{\prime}=\left\{1-\frac{T V(\Delta \alpha)^{2}}{\Delta C_{p} \Delta \beta}\right\} \Delta \beta=\left(\Delta C_{p}^{\text {intra }} / \Delta C_{p}\right) \Delta \beta \geq 0
$$

Since $\left(\mathrm{d} S^{\mathrm{c}}\right)_{\mathrm{g}}=0$ in the present case, performing the integration in eq 30 along the glass transition point by the use of eq 21 , we derive,

$$
\begin{aligned}
\Delta H & =-\int_{\mathrm{I}}^{\mathrm{II}} P V \mathrm{~d} \ln \tilde{V}+P_{0}\left(\tilde{V}_{\mathrm{g}}-\tilde{V}_{\mathrm{g} 0}\right) V_{0}^{*} \\
& \cong \tilde{V}\left(P_{\mathrm{g}}-3 P_{0}\right)(1-d) / 2
\end{aligned}
$$

Iso-Configurational Energy. When the assumption of the iso-configurational energy (iso- $U^{\mathrm{c}}$ ) as $T_{\mathrm{g}}$ is valid, we obtain, from eq 19,

$$
\left(\partial T_{\mathrm{g}} / \partial P\right)=(T V \Delta \alpha-P V \Delta \beta) /\left(\Delta C_{p}-P V \Delta \alpha\right)
$$

By the use of eq 6 and 7, eq 45, is transformed to

$$
\left(\frac{\partial T_{\mathrm{g}}}{\partial P}\right)=\frac{T V \Delta \alpha}{\Delta C_{p}}\left\{1-\frac{\Delta C_{p}^{\text {intra }} \times P V}{\Delta C_{p}^{\text {inter }}\left(\Delta C_{p} / \Delta \alpha-P V\right)}\right\}
$$

From eq 22, 23, and 45 with eq 6 and 7, we have

$$
\begin{aligned}
\alpha^{\prime} & =\left\{\frac{\Delta C_{p} \Delta \beta}{T V(\Delta \alpha)^{2}}-1\right\} \Delta \alpha /\left(1-\frac{P \Delta \beta}{T \Delta \alpha}\right) \\
& =\frac{\Delta C_{p}^{\text {intra }} \Delta \alpha}{\Delta C_{p}^{\text {inter }}(1-P \Delta \beta / T \Delta \alpha)} \\
\beta^{\prime} & =\left\{1-\frac{T V(\Delta \alpha)^{2}}{\Delta C_{p} \Delta \beta}\right\} \Delta \beta /\left(1-\frac{P V \Delta \alpha}{\Delta C_{p}}\right) \\
& =\frac{\Delta C_{p}^{\text {intra }} \Delta \beta}{\Delta C_{p}\left(1-P V \Delta \alpha / \Delta C_{p}\right)}
\end{aligned}
$$

The enthalpy difference $\Delta H$ is readily evaluated 
from eq 29 with $\left(\mathrm{d} U^{\mathrm{c}}\right)_{\mathrm{g}}=0$ as

$$
\Delta H=-P_{0} \tilde{V}_{\mathrm{g} 0} V_{0}^{*}(1-d)
$$

Iso-Configurational Enthalpy. From eq 20 with $\left(\partial H^{\mathrm{c}} / \partial P\right)_{\mathrm{g}}=0$, we can derive

$$
\left(\partial T_{\mathrm{g}} / \partial P\right)=\left\{T V \Delta \alpha-\left(V-V^{*}\right)\right\} / \Delta C_{p}<T V \Delta \alpha / \Delta C_{p}
$$

for the case of the iso-configurational enthalpy (iso- $H^{\mathrm{c}}$ ) assumption. Combining eq 50 and 22 or 23 , we obtain

$$
\alpha^{\prime}=\left\{\frac{\Delta \beta \Delta C_{p}}{T V(\Delta \alpha)^{2}-\left(V-V^{*}\right) \Delta \alpha}-1\right\} \Delta \alpha
$$

when $T V \Delta \alpha \neq\left(V-V^{*}\right)$, and

$$
\beta^{\prime}=\left\{1-\frac{T V(\Delta \alpha)^{2}-\left(V-V^{*}\right) \Delta \alpha}{\Delta C_{p} \Delta \beta}\right\} \Delta \beta>0
$$

From eq 31 and 21 with $\left(\mathrm{d} H^{\mathrm{c}}\right)_{\mathrm{g}}=0$, we have the expression for $\Delta H$ as

$$
\begin{aligned}
\Delta H= & -\int_{\mathrm{I}}^{\mathrm{II}}\left\{\left(P_{\mathrm{g}}-P_{0}\right) V \mathrm{~d} \ln \tilde{V}+\left(V-V^{*}\right) \mathrm{d} P\right\} \\
= & -\int_{\mathrm{I}}^{\mathrm{II}}\left[\left(P_{\mathrm{g}}-P_{0}\right) V \mathrm{~d} \ln \tilde{V}\right. \\
& \left.+\left\{\left(\tilde{V}_{\mathrm{g} 0}-1\right) V_{\mathrm{g} 0}^{*}+V\left(P-P_{0}\right)\left(\frac{\partial \ln \tilde{V}}{\partial P}\right)_{\mathrm{g}}\right\} \mathrm{d} P\right] \\
& \cong\left\{V(1-d)-V_{\mathrm{g} 0}^{*}\left(\tilde{V}_{\mathrm{g} 0}-1\right)\right\}\left(P_{\mathrm{g}}-P_{0}\right) \\
& \cong-\left(\tilde{V}_{\mathrm{g}}-1\right) V_{0}^{*}\left(P_{\mathrm{g}}-P_{0}\right)<0
\end{aligned}
$$

Adam-Gibbs Theory. Adam and Gibbs ${ }^{7}$ proposed a molecular kinetic theory which explains the temperature dependence of relaxational behavior in glass-forming liquids. The final result of their theory can be written as

$$
\tau=A \exp \left(C / T S^{\mathrm{c}}\right)
$$

where $\tau$ is the relaxation time, and $A$ and $C$ are constants. According to eq 54, the glass transition may occur at iso- $\left(T S^{\mathrm{c}}\right)$, i.e., $\left(\mathrm{d} T S^{\mathrm{c}}\right)_{\mathrm{g}}=$ $T\left(\mathrm{~d} S^{\mathrm{c}}\right)_{\mathrm{g}}+S_{\mathrm{g}}{ }^{\mathrm{c}}(\mathrm{d} T)_{\mathrm{g}}=0$. In this case, from eq 18 , 22 , and 23 with eq 6 and 7 , we have

$$
\begin{aligned}
\left(\partial T_{\mathrm{g}} / \partial P\right) & =T V \Delta \alpha /\left(\Delta C_{p}+S_{\mathrm{g}}{ }^{\mathrm{c}}\right) \\
& =T V \Delta \alpha /\left\{\Delta C_{p}(1+n)\right\}<T V \Delta \alpha / \Delta C_{p} \\
\alpha^{\prime} & =\left\{\frac{\Delta C_{p} \Delta \beta(1+n)}{T V(\Delta \alpha)^{2}}-1\right\} \Delta \alpha \\
& =\left\{\frac{\Delta C_{p}(1+n)}{\Delta C_{p}^{\text {tnter }}}-1\right\} \Delta \alpha
\end{aligned}
$$

$$
\begin{aligned}
\beta^{\prime} & =\left\{1-\frac{T V(\Delta \alpha)^{2}}{\Delta C_{p} \Delta \beta(1+n)}\right\} \Delta \beta \\
& =\left\{1-\frac{\Delta C_{p}^{\text {inter }}}{\Delta C_{p}(1+n)}\right\} \Delta \beta
\end{aligned}
$$

where $n=S_{\mathrm{g}}^{\mathrm{c}} / \Delta C_{p}$.

Putting $\left(\mathrm{d} T S^{\mathrm{c}}\right)_{\mathrm{g}}=0$ into eq 30 , we find the expression of the enthalpy $\Delta H$ represented by

$$
\begin{aligned}
\Delta H & =-n \Delta C_{p} \Delta T_{\mathrm{g}}+V\left(P_{\mathrm{g}}-P_{0}\right)(1-d) / 2 \\
& \cong\{-n T V \Delta \alpha /(1+n)+V(1-d) / 2\}\left(P_{\mathrm{g}}-P_{0}\right)
\end{aligned}
$$

\section{DISCUSSION}

\section{Criteria for Glass Transition}

In order to survey the theoretical predictions for $\left(\partial T_{\mathrm{g}} / \partial P\right), \alpha^{\prime}$ or $\beta^{\prime}$ and $\Delta H$ derived from each criterion for the glass transition, we first evaluate a few quantities involved in the expressions for them. Since internal pressure $P_{\mathrm{i}} \equiv(\partial U / \partial V)_{T}$ is expressed by $\boldsymbol{P}_{\mathrm{i}}=\boldsymbol{T} \alpha / \beta-P$, we have, using eq 6 ,

$$
\begin{aligned}
\Delta C_{p} / V \Delta \alpha \geq \Delta C_{p}^{\text {inter }} / V \Delta \alpha & =T \Delta \alpha / \Delta \beta \\
& =P_{\mathrm{i} 1}+\Delta P_{\mathrm{i}} \beta_{\mathrm{g}} / \Delta \beta+P \\
& \equiv P_{\mathrm{i}}{ }^{\mathrm{h}}+P
\end{aligned}
$$

where $P_{\text {il }}$ is $P_{\mathrm{i}}$ in the liquid state, $\Delta P_{\mathrm{i}}$ is the difference in $P_{\mathrm{i}}$ between the liquid and glassy states, and $P_{\mathrm{i}}{ }^{\mathrm{h}} \equiv \boldsymbol{P}_{\mathrm{i} 1}+\Delta \boldsymbol{P}_{\mathrm{i}} \beta_{\mathrm{g}} / \Delta \beta$. As seen from eq $3^{\prime}, 8$ and $59, P_{\mathrm{i}}^{\mathrm{h}}$ is equal to $\left(\partial U_{2} / \partial \tilde{V}\right)_{V^{*}, T} / V^{*}$ and may be positive in any range of $\tilde{V}$, i.e.,

$$
P_{\mathrm{i}}{ }^{\mathrm{h}}=P_{\mathrm{i} 1}+\Delta P_{\mathrm{i}} \beta_{\mathrm{g}} / \Delta \beta=\left(\partial U_{2} / \partial \tilde{V}\right)_{V^{*}, T} / V^{*}>0
$$

The value of $\Delta P_{\mathrm{i}}$ is about $60-70 \%$ of $P_{\mathrm{i} 1},{ }^{10}$ and the ratio of $\beta_{\mathrm{g}} / \Delta \beta$ is about $1-2$ at atmospheric pressure in amorphous polymers. ${ }^{10}$ Accordingly, $\boldsymbol{P}_{\mathrm{i}}{ }^{\mathrm{h}}$ may be estimated as about $5000-9000$ atm from the definition of eq 60 , since $P_{\mathrm{i} 1}$ is usually $3000-4000$ atm in the vicinity of the glass transition point at atmospheric pressure.

On the other hand, the value of $V-V^{*}$ appearing in the expressions for the iso- $H^{\mathrm{c}}$ may be the same order of $T V \Delta \alpha$ because $V$ and $V^{*}$ may be approximated by $V=V_{0}+V \alpha_{1} T$ and $V^{*}=V_{0}+V_{\alpha_{\mathrm{g}}} T$, respectively, where $V_{0}$ is the volume of hypothetical liquid at $0^{\circ} \mathrm{K}$, and $\alpha_{1}$ is thermal expansion coefficient in the liquid 
A Hole Theory of Polymer Lixuids and Glasses. IV

Table I. Characteristic values for glass transition

\begin{tabular}{|c|c|c|c|c|c|c|c|c|c|}
\hline Polymer & $\underset{\circ}{T_{\mathrm{g}},}$ & $\begin{array}{l}V_{\mathrm{g}}, \\
\mathrm{cc} / \mathrm{g}\end{array}$ & $\begin{array}{l}\Delta \alpha \times \\
10^{-4}, \\
\operatorname{deg}^{-3}\end{array}$ & $\begin{array}{r}\Delta \beta \times \\
10^{-5} \\
\text { atm }^{-1}\end{array}$ & $\begin{array}{c}\Delta C_{p} \\
\text { cal/deg g }\end{array}$ & $\begin{array}{r}\partial T_{\mathrm{g}} / \partial P \\
\mathrm{deg} / \mathrm{atm}\end{array}$ & $\frac{T V \Delta \alpha}{\Delta C_{p}}$ & $\begin{array}{c}\frac{\Delta \beta}{\Delta \alpha}, \\
\operatorname{deg} / \operatorname{atm}\end{array}$ & $\frac{T V \Delta \alpha}{\Delta C_{p}(1+n)}$ \\
\hline Polystyrene & $362^{\mathrm{a}}$ & $0.97^{\mathrm{a}}$ & $3.0^{\mathrm{a}}$ & $1.56^{\mathrm{a}}$ & $0.075^{b}$ & $0.030^{a}$ & 0.034 & 0.052 & 0.029 \\
\hline Poly(methyl methacrylate) & $384^{\mathrm{a}}$ & $0.85_{5}^{\mathrm{a}}$ & $2.9^{a}$ & $1.23^{\mathrm{a}}$ & $0.086^{\mathrm{e}}$ & $0.023^{a}$ & 0.027 & $0 . .42$ & 0.024 \\
\hline Poly(vinyl chloride) & $353^{\mathrm{a}}$ & $0.75^{\mathrm{a}}$ & $2.15^{\mathrm{a}}$ & $0.89^{d, a}$ & $0.068^{e}$ & $0.013_{5}{ }^{a}$ & $0.020_{3}$ & 0.041 & 0.0172 \\
\hline Poly(vinyl acetate) & $298^{\mathrm{f}}$ & $0.855^{\mathrm{f}}$ & $4.0^{f}$ & - & $0.10^{f}$ & $0.022^{\mathrm{f}}$ & 0.025 & 0.0393 & $0.020_{5}$ \\
\hline Polyisobutylene & $198^{f}$ & $1.048^{\mathrm{f}}$ & $4.5^{f}$ & $2.5^{\mathrm{h}}$ & $0.09^{f}$ & $0.024^{f}$ & 0.024 & 0.053 & 0.017 \\
\hline Natural rubber & $252^{\mathrm{i}}$ & $0.96^{\mathrm{i}}$ & $4.4^{i}$ & $1.21^{j}$ & $(0.125)^{\mathrm{k}}$ & $0.024^{j}$ & $\left(0.020_{5}\right)$ & $0.027_{5}$ & $\longrightarrow$ \\
\hline
\end{tabular}

a ref $12, \mathrm{~b}$ ref $13, \mathrm{c}$ ref $14, \mathrm{~d} \operatorname{ref} 4$, e ref $15, \mathrm{f}$ ref $16, \mathrm{~g}$ ref $17, \mathrm{~h}$ ref 18 , i ref $19, \mathrm{j}$ ref 20 ,

$\mathrm{k}$ ref 21 .

state. In fact, $\left(V-V^{*}\right) / V$ at $T_{\mathrm{g}}$ under atmospheric pressure is estimated to be about 0.085 from the analysis in the preceding paper, ${ }^{9}$ whereas $T_{\mathrm{g}} \Delta \alpha$ is about 0.113 according to Simha and Boyer $^{11}$ in amorphous polymers.

The value of $n\left(\equiv S_{\mathrm{g}}{ }^{\mathrm{c}} / \Delta C_{p}\right)$ involved in the theoretical results obtained from Adam-Gibbs theory may be approximated by $\ln \left(T_{\mathrm{g}} / T_{2}\right)$ because $S_{\mathrm{g}}^{\mathrm{c}}=\int_{T_{2}}^{T_{\mathrm{g}}} \Delta C_{p} / T \mathrm{~d} T \cong \Delta C_{p} \ln \left(T_{\mathrm{g}} / T_{2}\right), \quad$ where $T_{2}$ is the temperature at which $S^{\mathrm{c}}$ would become zero. Therefore, since $T_{\mathrm{g}} / T_{2}=1.30( \pm 8.4 \%)$ for glass-forming liquids according to the analysis of Adam and Gibbs, ${ }^{7}$ the value of $n$ is estimated at about 0.26 .

As seen from eq 41 and 45 with eq 59 and 60, $\left(\partial T_{\mathrm{g}} / \partial P\right)$ for the iso- $U^{\mathrm{c}}$ is smaller than that for the iso- $S^{\mathrm{c}}$, and therefore $\alpha^{\prime}$ or $\beta^{\prime}$ for the iso- $U^{\mathrm{c}}$ is larger than that for the iso- $S^{\mathrm{c}}$. However, when the pressure is not so high, i.e., $P \ll P_{\mathrm{i}}{ }^{\mathrm{h}}+P$, eq 45 , 47 , and 48 for the iso- $U^{\mathrm{c}}$ become identical with the corresponding equations for the iso- $S^{\mathrm{c}}$, i.e., eq 41,42 , and 43 , as also seen from eq 59 . Therefore the iso- $U^{\mathrm{c}}$ is equivalent to the iso- $S^{\mathrm{c}}$ when $P \ll P_{\mathrm{i}}{ }^{\mathrm{h}}+P$ as far as the pressure dependence of the glass transition temperature is concerned. It follows also from eq 59 that $\Delta H$ for the iso$S^{\mathrm{c}}$ expressed by eq 44 is negligibly small compared with $\Delta H^{\text {inter }}$ given by eq 34 under low pressure. Accordingly when $P \ll P_{\mathrm{i}}{ }^{\mathrm{h}}+P, \Delta H \cong 0$ which ts identical with the result for the iso- $U^{\mathrm{c}}$ represented by eq 49 . As for the iso- $H^{c}$, since $V-V^{*}$ is the same order of $T V \Delta \alpha$, the pressure dependence of $T_{\mathrm{g}},\left(\partial T_{\mathrm{g}} / \partial P\right)$, expressed by eq 50 is much smaller than that for the iso- $S^{\circ}$ (eq 41), and thus $\beta^{\prime}$ is much larger, as seen from eq 52 , and 43 .

On the other hand, the Adam-Gibbs theory predicts that the value of $\left(\partial T_{\mathrm{g}} / \partial P\right)$ is a little smaller than that for the iso- $S^{\circ}$, as indicated by comparing eq 55 with eq 41 , because $n$ is about 0.26 . The enthalpy difference $\Delta H$ shows a negative but a small deviation from that of the iso- $S^{\mathrm{c}}$.

To summarize the theoretical predictions in $P \ll P_{\mathrm{i}}^{\mathrm{h}}+P$ : as for $\left(\partial T_{\mathrm{g}} / \partial P\right)$

$$
\begin{aligned}
\left(\text { iso- } H^{\mathrm{c}}\right) & <\left(\text { iso- } T S^{\mathrm{c}}\right)=T V \Delta \alpha /\left\{\Delta C_{p}(1+n)\right\}<\left(\text { iso- } U^{\mathrm{c}}\right) \\
& \cong\left(\text { iso- } S^{\mathrm{c}}\right)=T V \Delta \alpha / \Delta C_{p} \\
& <\Delta \beta / \Delta \alpha=\text { iso-free volume })
\end{aligned}
$$

as for $\beta^{\prime}$

$$
\begin{aligned}
\left(\text { iso- } H^{\mathrm{c}}\right) & >\left(\text { iso- } T S^{\mathrm{c}}\right)>\left(\text { iso- } U^{\mathrm{c}}\right) \cong\left(\text { iso- } S^{\mathrm{c}}\right) \\
& =\left(\Delta C_{p}^{\text {intra }} / \Delta C_{p}\right) \Delta \beta \\
& \geq 0=(\text { iso-free volume })
\end{aligned}
$$

as for $\Delta H$

$$
\begin{aligned}
\left(\text { iso- } H^{\mathrm{c}}\right) & <\left(\text { iso- } T S^{\mathrm{c}}\right)=-n \Delta C_{p} \Delta T_{\mathrm{g}} \\
& <\left(\text { iso- } U^{\mathrm{c}}\right)=0 \cong\left(\text { iso- } S^{\mathrm{c}}\right) \\
& <\Delta \bar{C}_{p}^{\text {intra }} \Delta T_{\mathrm{g}}=(\text { iso-free volume })
\end{aligned}
$$

and as for $\partial \Delta H / \partial P_{\mathrm{g}}$

$$
\begin{aligned}
\left(\text { iso- } H^{\mathrm{c}}\right) & <\left(\text { iso- } T S^{\mathrm{c}}\right) \\
& =-n T V \Delta \alpha /(1+n)<\left(\text { iso- } U^{\mathrm{c}}\right)=0 \\
& \cong\left(\text { iso- } S^{\mathrm{c}}\right)<\Delta C_{p}^{\text {intra }} \Delta \beta / \Delta \alpha \\
& =(\text { iso-free volume })
\end{aligned}
$$




\section{T. Nose}

Here eq 64 is derived from eq 37 with eq 59 and 61 .

In Table I, we show characteristic values for the glass transition calculated from published data near atmospheric pressure. The values of $n$ required in the calculation of the last column of Table $I$ are those obtained by Adam and Gibbs. $^{7}$ The values of $\left(\partial T_{\mathrm{g}} / \partial P\right)$ are almost equal to those of $T V \Delta \alpha / \Delta C_{p}$ or $T V \Delta \alpha /\left\{\Delta C_{p}(1+n)\right\}$, and much smaller than those of $\Delta \beta / \Delta \alpha$. This fact supports the assumption of the iso- $S^{\mathrm{c}}$, the iso- $U^{\mathrm{c}}$, or the iso- $T S^{\mathrm{c}}$, not that of the iso-free volume or the iso- $H^{\mathrm{c}}$, as seen from eq 61 . It appears that the iso- $T S^{\mathrm{c}}$, Adam-Gibbs theory (eq 55), shows closer agreement with experimental observations than the iso- $S^{\mathrm{c}}$ (eq 41). However, the difference between predicted values from these two criteria is so small that we cannot make a definite conclusion on this point, because the data in Table I were collected from the results obtained by different workers and made for different samples. In any case it is clear that the glass transition occurs near the iso- $S^{\mathrm{c}}$, the iso- $U^{\mathrm{c}}$, or the iso- $T S^{\mathrm{c}}$ rather than at the iso-free volume.

Upon cooling a liquid amorphous polymer under elevated pressure, densified glass is formed. This fact indicates that $\alpha^{\prime}, \beta^{\prime}>0$, in other words $\left(\partial T_{\mathrm{g}} / \partial P\right)<\Delta \beta / \Delta \alpha$ because of eq 22 and 23 . Gee ${ }^{4}$ has already presented an equation equivalent to eq 22 with a less general method of derivation which has shown close agreement with experimental results. The formation of such densified glasses indicates that the iso-free volume at $T_{\mathrm{g}}$ is not a valid assumption.

The iso- $S^{\mathrm{c}}$, the iso- $U^{\mathrm{c}}$, or the iso- $T S^{\mathrm{c}}$ concept is also verified by the experimental fact that the densified glass of polystyrene has almost the same enthalpy as the nondensified glass (see eq 63), which will be discussed in more detail in the next section.

\section{Enthalpy of Glass}

In the case of densified glass, we can see $\Delta H^{\text {inter }}<0$ and $\Delta H^{\text {intra }}>0$ from eq 32 and 35 , since $d<1$ and $\Delta T_{\mathrm{g}}>0$. Namely, intersegmental interactions make a negative contribution to the enthalpy difference $\Delta H$ because the densified glass has less fraction of frozen holes, whereas intrasegmental ones make a positive contribution because the chain conformation of the densified glass is frozen in a higher temperature, i.e., in a higher energy state. This qualitative interpretation has been already proposed by Allen, et $a{ }^{25}{ }^{25}$ When the iso- $U^{\mathrm{c}}$, the iso- $S^{\mathrm{c}}$, or the iso- $T S^{c}$ is a valid assumption as suggested in the preceding section, $\Delta H$ should be nearly equal to zero from eq 63 , i.e., $\Delta H^{\text {inter }}=-\Delta H^{\text {intra }}$.

On the other hand, the annealed glass of higher density formed at a slow cooling rate under atmospheric pressure should show different behavior, because the annealed glass is formed at a temperature lower than the glass forming temperature of the densified glass. In the case of annealed glass, from eq 21, we have $-(1-d)=\Delta \bar{\alpha} \Delta T_{\mathrm{g}}$. Accordingly enthalpy difference $\Delta H$ is given, with the use of eq $32^{\prime}$ and 35 , by

$$
\begin{aligned}
\Delta H & =\Delta \bar{C}_{p}^{\text {intra }} \Delta T_{\mathrm{g}}-\Delta \bar{C}_{p}^{\text {inter }}(1-d) / \Delta \bar{\alpha} \\
& =\Delta \bar{C}_{p} \Delta T_{\mathrm{g}} \\
& =-\Delta \bar{C}_{p}(1-d) \Delta \bar{\alpha}
\end{aligned}
$$

In the annealed glass, the difference in glass forming temperature, $\Delta T_{\mathrm{g}}$, is negative, whereas $1-d$ is positive. Therefore both inter- and intrasegmental interactions, i.e., both the hole fraction and chain conformation, make nagative contributions to $\Delta H$. In other words, it is found that the annealed glass is expected to have lower enthaly by the amount given in eq 65 .

When the densified glass is heated under atmospheric pressure at an ordinary heating rate, the volume increases sharply in the vicinity of $T_{\mathrm{g}},{ }^{22}$ as is observed in the annealed glass obtained by a slow cooling rate. On the other hand, heat absorption or a peak of heat capacity in the densified glass is not observed in the vicinity of $T_{g}$, as is found in the annealed glass. ${ }^{23,24}$ Corresponding to this fact, it has been found by measurements of heat of the solution that the densified glass has almost the same enthalpy as glass formed under ordinary conditions, ${ }^{23,25}$ whereas the annealed glass has a lower value of enthalpy. ${ }^{23}$ These experimental facts are precisely those predicted by this theory for annealed glass (eq 65) and the desified glass with the iso- $S^{3}$, the iso- $U^{\mathrm{c}}$, or the iso- $T S^{\mathrm{c}}$ criterion (eq 63).

Ichihara, et al. ${ }^{23}$ obtained the result that $\Delta H$ 
for the densified polystyrene is zero in the pressure range of measurements, $0-600 \mathrm{~atm}$, and Allen, et al.,$^{25}$ found that $\Delta H$ for the densified polystyrene glass formed at $1250 \mathrm{~atm}$ is negative but very small.

The eq 65 for the annealed glass is quantitatively satisfied by experiments ${ }^{23}$ for polystyrene and $\operatorname{poly}(\alpha$-methylstyrene).

As can be seen from eq 28, if we measure the difference in enthalpy between the annealed and densified glasses which have the same specific volume, i.e., the same $\tilde{V}_{\mathrm{g}}$, we may obtain the difference in $U_{1}^{\mathrm{c}}$ between different temperatures because the glass forming temperature of the densified glass is higher than that of the annealed glass, then we may evaluate $\Delta C_{p}^{\text {intra }}$, and separate $\Delta C_{p}$ into $\Delta C^{\text {inter }}$ and $\Delta C_{p}^{\text {intra. }}$. It should be noticed, however, that this method is essentially based on the present model with Assumption 1, 2 , and 3. (Alternatively, we may estimate $\Delta C_{p}^{\text {inter }}$ from eq 6 as already presented in the preceding paper. ${ }^{8}$ )

Role of Intrasegmental Interactions in Microbrownian Motion

When $\Delta C_{p}^{\text {intra }}=0$ or when there is no intrasegmental degrees of freedom, the iso-free volume, the iso- $S^{\mathrm{c}}$, and the iso- $U^{\mathrm{c}}$ are equivaent to each other, as seen from eq 9 and 11 . Then $\left(\partial T_{\mathrm{g}} / \partial P\right)$ $=T V \Delta \alpha / \Delta C_{p}=\Delta \beta / \Delta \alpha$, and $\alpha^{\prime}=\beta^{\prime}=0$ for any one of the criteria. Densified glass would not therefore formed. This is also readily seen from eq $6,7,41,42,43,45,47$ and 48 with $\Delta C_{p}^{\text {intra }}=0$. Accordingly the observed fact $T V \Delta \alpha / \Delta C_{p}<\Delta \beta / \Delta \alpha$ and the phenomenon of formation of densified glass may be closely related to the conformation of chain backbone. The satisfaction of iso- $S^{\mathrm{c}}$ (or iso- $U^{\mathrm{c}}$ ) at $T_{\mathrm{g}}$ suggests that the intrasegmental interaction or the flexibility of a chain molecule also plays as important a role in the segmental motion as the free volume ${ }^{10}$ and that the segmental motion can have the same mobility in the less free volume when the chain is more flexible. Thus the glass transition occurs at a smaller value of $\tilde{V}$ under elevated pressure, since the chain is more flexible at a higher temperature. This causes the formation of the densified glass having a lesser fraction of frozen holes. The importance of molecular flexibility is also suggested by the following fact, as pointed out in the previous paper: ${ }^{9}$ the free-volume fraction $\left(1-V_{0} / V_{\mathrm{g}}\right)$ at $T_{\mathrm{g}}$ decreases from about 0.17 for high-molecular-weight polymers at about 0.05 for simple low-molecular-weight substances with decreases in the chain length, where $V_{0}$ is the volume of a hypothetical liquid at $0^{\circ} \mathrm{K}$ and $V_{\mathrm{g}}$ is the volume at $T_{\mathrm{g}}$.

Acknowlegments. The author wishes to thank Prof. T. Hata of the Tokyo Institute of Technology and Dr. S. Ichihara for encouraging discussions and much helpful advice.

\section{APPENDIX}

In order to see the relationship between eq 5 and Assumption 1, we will evaluate from eq 5 the change in the cell volume with temperature and pressure in the liquid and glassy states, and that with frozen-hole fraction of the glass.

Inserting eq 5 into eq 2 and 4 , and differentiating both sides of the obtained equations with temperature (or pressure) under constant pressure (or temperature), we have

$$
\begin{gathered}
P V \alpha_{1}+\left[\frac{\partial^{2} F_{2}^{\prime \prime}}{\partial\left(\ln V^{*}\right)^{2}}\right]_{T} \alpha_{1}^{*}+\left[\frac{\partial}{\partial T}\left(\frac{\partial F_{2}^{\prime \prime}}{\partial \ln V^{*}}\right)_{T}\right]_{V *}=0 \\
P V \alpha_{\mathrm{g}}+\left[\frac{\partial^{2} F_{2}^{\prime \prime}}{\partial\left(\ln V^{*}\right)^{2}}\right]_{T} \alpha_{\mathrm{g}}{ }^{*}+\left[\frac{\partial}{\partial T}\left(\frac{\partial F_{2}^{\prime \prime}}{\partial \ln V^{*}}\right)_{V}\right]_{V^{*}}=0 \\
V\left(1+P \beta_{1}\right)-\left[\frac{\partial^{2} F_{2}^{\prime \prime}}{\partial\left(\ln V^{*}\right)^{2}}\right]_{T} \beta_{1}^{*}=0 \quad(\mathrm{~A}-2) \\
V\left(1+P \beta_{\mathrm{g}}\right)-\left[\frac{\partial^{2} F_{2}^{\prime \prime}}{\partial\left(\ln V^{*}\right)^{2}}\right]_{T} \beta_{\mathrm{g}}{ }^{*}=0
\end{gathered}
$$

where $\alpha=(\partial \ln V / \partial T)_{p}, \quad \beta=-(\partial \ln V / \partial P)_{T}, \quad \alpha^{*}$ $=\left(\partial \ln V^{*} / \partial T\right)_{p}$, and $\beta^{*}=-\left(\partial \ln V^{*} / \partial P\right)_{T}$, and the subscripts 1 and $g$ refer to the liquid and glassy states, respectively. It must be noted here that $\alpha_{\mathrm{g}}{ }^{*}=\alpha_{\mathrm{g}}$ and $\beta_{\mathrm{g}}{ }^{*}=\beta_{\mathrm{g}}$ because $\tilde{V}$ is constant in the glassy state. From eq A-1, A-2, A-3, and A-4, we obtain

$$
\left(\alpha_{1}{ }^{*}-\alpha_{\mathrm{g}}{ }^{*}\right) /\left(\alpha_{1}-\alpha_{\mathrm{g}}\right)=\boldsymbol{P} \beta_{\mathrm{g}} /\left(1+\boldsymbol{P} \beta_{\mathrm{g}}\right)
$$

and

$$
\left(\beta_{1}{ }^{*}-\beta_{\mathrm{g}}{ }^{*}\right) /\left(\beta_{1}-\beta_{\mathrm{g}}\right)=P \beta_{\mathrm{g}} /\left(1+P \beta_{\mathrm{g}}\right)
$$

at glass transition. Therefore, under $P \beta_{g} /(1+$ $\left.P \beta_{\mathrm{g}}\right) \ll 1, \alpha_{1}{ }^{*}=\alpha_{\mathrm{g}}{ }^{*}$ and $\beta_{1}{ }^{*}=\beta_{\mathrm{g}}{ }^{*}$, which are what Assumption 1 means. 
We now consider the cell volume in the two different glasses having different frozen-hole fractions, $1-\tilde{V}_{1}^{-1}$ and $1-\tilde{V}_{2}^{-1}$. It follows from eq 2 and A-4 that

$$
P V_{1}+\left.\left(\partial F_{2}^{\prime \prime} / \partial \ln V^{*}\right)_{T}\right|_{1_{1}^{*}}=0
$$

and

$$
P V_{2}+\left.\left(\partial F_{2}^{\prime \prime} / \partial \ln V^{*}\right)_{T}\right|_{V_{2}}=0
$$

where the subscripts 1 and 2 refer to two different glasses, and the symbol $\left.\right|_{V_{i}}$ designates the value at $V_{i}^{*}$. Since

$$
\begin{aligned}
\left.\left(\partial F_{2}{ }^{\prime \prime} / \partial \ln V^{*}\right)_{T}\right|_{V_{2}{ }^{*}}-\left.\left(\partial F_{2}{ }^{\prime \prime} / \partial \ln V^{*}\right)_{T}\right|_{V_{1}{ }^{*}} \\
\cong\left[\partial^{2}{F_{2}}^{\prime \prime} / \partial\left(\ln V^{*}\right)^{2}\right]_{T}\left(\ln V_{2}{ }^{*}-\ln V_{1}{ }^{*}\right),
\end{aligned}
$$

we can derive from eq A-7, A-8, and A-4,

$$
\left(V_{2}^{*} / V_{1}^{*}-1\right) /\left(\tilde{V}_{2} / \tilde{V}_{1}-1\right) \cong-P \beta_{\mathrm{g}} /\left(1+P \beta_{\mathrm{g}}\right)
$$

Therefore, under $P \beta_{\mathrm{g}} /\left(1+P \beta_{\mathrm{g}}\right) \ll 1, \quad V_{2}{ }^{*}=V_{1}{ }^{*}$, which is again what Assumption 1 means. Likewise, we can derive from eq A-2 and A-4

$$
\alpha_{\mathrm{g} 2} / \alpha_{\mathrm{g} 1} \cong 1
$$

and

$$
\beta_{\mathrm{g} 2} / \beta_{\mathrm{g} 2} \cong \tilde{V}_{2} / \tilde{V}_{1}
$$

\section{LIST OF SYMBOLS}

$A$, constant in Adam-Gibbs' equation (eq 54)

$C$, constant in Adam-Gibbs' equation (eq 54)

$\Delta C_{p}$, difference in heat capacity between the liquid and glassy states

$\Delta \bar{C}_{p}$, mean value of difference in heat capacity between liquid ahd glassy states, equal to $\Delta \bar{C}_{p}^{\text {inter }}+\Delta \bar{C}_{p}^{\text {intra }}$

$\Delta \bar{C}_{p}^{\text {inter }}$, mean value of $\Delta C_{p}^{\text {inter }}$ in the temperature range between $T_{\mathrm{g} 0}$ and $T_{\mathrm{g} 0}-$ $(1-d) / \Delta \bar{\alpha}$

$\Delta \bar{C}_{p}^{\text {intra }}$, mean value of $\Delta C_{p}^{\text {intra }}$ in the temperature range between $T_{\mathrm{g}}$ and $T_{\mathrm{g} 0}$

$d$, ratio of reduced volume $\tilde{V}$ of densified glass to nondensified glass, $\widetilde{V}_{\mathrm{g}} / \widetilde{V}_{\mathrm{g} 0}$

$F$, Helmholtz free energy

$F_{2}{ }^{\prime}$, part of Helmholtz free energy which is a function of $\tilde{V}$ and $T$ (eq 5)

$F_{2}{ }^{\prime \prime}$, part of Helmholtz free energy which is a function of $V^{*}$ and $T$ (eq 5)

$H^{\mathrm{c}}$, configurational enthalpy defined by eq 14
$\Delta H$, enthalpy difference between two glasses when the glass formed at an ordinary cooling rate under atmospheric pressure is taken as the reference

inter, superscript for intersegmental interactions

intra, superscript for intrasegmental interactions

$M$, total number of lattice sites in the system

$N$, total number of segments in the system

$n$, ratio of configurational entropy to the difference in heat capacity at $T_{\mathrm{g}}, S_{\mathrm{g}}^{\mathrm{c}} /$ $\Delta C_{p}$

$P$, pressure

$\boldsymbol{P}_{0}$, atmospheric pressure at which the reference glass is formed

$P_{\mathrm{g}}$, pressure at glass transition point

$\boldsymbol{P}_{\mathrm{i}}$, internal pressure, $(\partial U / \partial V)_{T}$

$\boldsymbol{P}_{\text {i1 }}$, internal pressure in the liquid state

$\boldsymbol{P}_{\mathrm{i}}{ }^{\mathrm{h}}$, quantity defined by $\boldsymbol{P}_{\mathrm{i}}{ }^{\mathrm{h}}=\boldsymbol{P}_{\mathrm{i} 1}+\Delta \boldsymbol{P}_{\mathrm{i}} \Delta \beta_{\mathrm{g}} /$ $\Delta \beta$ (eq 60)

$\Delta P_{\mathrm{i}}$, difference of internal pressure between the liquid and glassy states

$S$, entropy

$S_{2}{ }^{\prime}$, part of entropy which is a function of $\tilde{V}$ and $T$ (eq 5)

$S_{2}{ }^{\prime \prime}$, part of entropy which is a function of $V^{*}$ and $T$ (eq 5)

$S^{\mathrm{c}}$, configurational entropy defined as a part of entropy which is frozen-in in the glassy state (eq 9)

$S_{1}$, entropy associated with the chain conformation which is not allowed to change in the glassy state

$S_{\text {g }}{ }^{\mathrm{c}}$, configurational entropy at glass transition temperature

$T$, temperature

$T_{0}$, temperature at which enthalpies of glasses are compared in the evaluation of $\Delta H$

$T_{2}$, temperature at which configurational entropy would become zero in the theory of Adam and Gibbs

$T_{\mathrm{g} 0}$, temperature at which the reference glass in the evaluation of $\Delta H$ is formed

$\Delta T_{\mathrm{g}}$, difference in glass transition temperature, $T_{\mathrm{g}}-T_{\mathrm{g} 0}$

$U$, internal energy 
A Hole Theory of Polymer Liquids and Glasses. IV

$U_{2}{ }^{\prime}$, part of internal energy which is a function of $\tilde{V}$ and $T$ (eq 5)

$U_{2}^{\prime \prime}$, part of internal energy which is a function of $V^{*}$ and $T$ (eq 5)

$U^{\mathrm{c}}$, configurational energy defined as a part of internal energy which is frozenin in the glassy state (eq 11)

$U_{1}{ }^{\mathrm{c}}$, internal energy associated with the chain conformation which is not allowed to change in the glassy state

$V$, volume

$\tilde{V}$, reduced volume defined as $\tilde{V}=V / V^{*}=$ $M / N$

$V^{*}$, volume of cells occupied by segments, $v^{*} N$

$V_{\mathrm{g}}$, volume at glass transition temperature

$V_{\mathrm{s}}$, volume of the glass measured at the same temperature and pressure (eq 26 and 27)

$V_{0}$, volume of hypothetical liquid at $0^{\circ} \mathrm{K}$, equal to $V^{*}$ at $0^{\circ} \mathrm{K}$

$\tilde{V}_{\text {g }}$, reduced volume $\tilde{V}$ at glass transition temperature

$V_{0}^{*}$, volume of cells $V^{*}$ at $\left(T_{0}, P_{0}\right)$

$\tilde{V}_{\mathrm{g} 0}$, reduced volume $\tilde{V}_{\mathrm{g}}$ at $T_{\mathrm{g}}$ of the reference glass in the evaluation of $\Delta H$

$v^{*}$, volume of a cell

$\alpha^{\prime}$, quantity defined by eq 24 or 26 , which represents the decrease in the frozen hole fraction with the increase of glass transition temperature under elevated pressure

$\alpha_{\mathrm{g}}$, thermal expansion coefficient in the glassy state

$\alpha_{1}$, thermal expansion coefficient in the liquid state

$\Delta \alpha$, difference in thermal expansion coefficient between the liquid and glassy states

$\Delta \bar{\alpha}$, mean value of $\Delta \alpha$ in the temperature range between $T_{\mathrm{g} 0}$ and $T_{\mathrm{g} 0}-(1-d) / \Delta \bar{\alpha}$

$\beta^{\prime}$, quantity defined by eq 25 or 27 , which represents the decrease in the frozen hole fraction with the increase in pressure of glass-formation

$\beta_{\mathrm{g}}$, isothermal compressibility in the glassy state

$\Delta \beta$, difference in isothermal compressibility between the liquid and glassy states $\tau$, relaxation time of microbrownian motion (eq 54)

1 , as subscript designates the intrasegmental interactions

2 , as subscript designates the intersemental interactions

$(\partial x / \partial y)_{g}$, change of $x_{g}, x$ at glass transition point, with $y$

$(\mathrm{d} x)_{\mathrm{g}}$, change of $x_{s}, x$ at glass transition point $(\mathrm{d} x)^{1}$, change of $x$ in the liquid state

$O(x)$, order of magnitude of $x$

\section{REFERENCES}

1. J. M. O'Reilly, "Modern Aspects of The Vitreous State," Vol. III, J. D. Mackenzie, Ed., Butterworths Scientific Publications Ltd., London, 1964, p 59.

2. M. Goldstein, J. Chem. Phys., 39, 3369 (1963).

3. N. Shiskkin, Soviet Physics Solid State, 2, 322 (1960).

4. G. Gee, Polymer, 7, 177 (1966).

5. e. g. J. D. Ferry, "Viscoelastic Properties of Polymers," John Wiley \& Sons, Inc., New York, N. Y., 1961.

6. J. H. Gibbs and E. A. DiMarzio, J. Chem. Phys., 28, 373, 807 (1958).

7. G. Adam and J. H. Gibbs, J. Chem. Phys., 43, 139 (1965).

8. T. Nose, Polymer J., 2, 437 (1971).

9. T. Nose, Polymer J., 2427 (1971).

10. T. Nose and T. Hata, "Proceedings of the 5 th International Congress on Rheology," Vol. 3, University of Tokyo Press, Tokyo, 1970, p 215.

11. R. Simha and R. F. Boyer, J. Chem. Phys., 37, 1003 (1962).

12. K. H. Hellwege, W. Knappe, and P. Lahmann, Kolloid-Z, 183, 110 (1962).

13. F. E. Karasz, H. E. Bair, and J. M. O'Reilly, J. Phys. Chem., 69, 2657 (1965).

14. J. M. O'Reilly, J. Polym. Sci., Part C, 14, 49 (1964).

15. B. Wunderlich, J. Phys. Chem., 64, 1052 (1960).

16. J. M. O'Reilly, J. Polym. Sci., 57, 429 (1962).

17. J. E. McKinney and H. V. Belcher, J. Res. Nat. Bur. Stand., 67A, 43 (1963).

18. H. Simgh and A. W. Nolle, J. Appl. Phys., 30, 337 (1959).

19. G. M. Martin and L. Mandelkern, J. Res. Nat. Bur. Stand., 62, 141 (1959).

20. J. E. McKinney, H. V. Belcher, and R. S. Marvin, Trans. Soc. Rheology, 4, 347 (1960).

21. N. Beckkedahl and R. B. Scott, J. Res. Nat. 
T. Nose

Bur. Stand., 29, 87 (1942).

22. N. Shishkin, ref 3; K. Tanaka, T. Nose and T. Hata, unpublished data.

23. S. Ichihara, A. Komatsu, and T. Hata, to be published in Polymer J., 2, 530 (1971).
24. S. I. Meeson and S. M. Lipatov, Colloid $J$. USSR, 21, 509 (1959).

25. G. Allen, R. C. Ayerst, J. R. Clevel, G. Gee, and C. Prices, J. Polym. Sci., Part C, 23, 127 (1968). 